\title{
Sterol regulatory element-binding protein 1 cooperates with c-Myc to promote epithelial-mesenchymal transition in colorectal cancer
}

\author{
DUANYANG ZHAI, CHUNHUI CUI, LANG XIE, LIANXU CAI and JINLONG YU \\ Department of General Surgery, Zhujiang Hospital of Southern Medical University, \\ Guangzhou, Guangdong 510530, P.R. China
}

Received July 6, 2017; Accepted February 8, 2018

DOI: $10.3892 / \mathrm{ol} .2018 .8058$

\begin{abstract}
Metastasis is the primary cause of mortality in colorectal cancer (CRC), the mechanism of which remains unclear. In the present study, by detecting mRNA expression using a reverse transcription-quantitative polymerase chain reaction (qPCR), it was revealed that sterol regulatory element-binding protein 1 (SREBP1) is highly expressed in CRC. Using a cell wound healing assay and a cell invasion assay, a novel metastasis-promoting role for SREBP1 in CRC was identified. Furthermore, snail family transcriptional repressor 1 (SNAIL) was identified as a key downstream effector of SREBP1 in CRC by the use of small interfering RNA against SNAIL. Additionally, using co-immunoprecipitation and chromatin immunoprecipitation-qPCR assays, it was demonstrated that SREBP1 interacts with c-MYC to enhance the binding of c-MYC to the promoter of the mesenchymal gene, SNAIL, thereby increasing SNAIL expression and accelerating epithelial-mesenchymal transition. These results indicated a novel role for SREBP1 and provide insight into the regulatory mechanisms of the c-Myc oncogene in $\mathrm{CRC}$, which may function as a potential therapeutic target for $\mathrm{CRC}$ treatment.
\end{abstract}

\section{Introduction}

Human colorectal cancer (CRC) is one of the most common types of cancer globally and one of the leading causes of cancer-associated mortality in developed countries (1). A large proportion of patients with CRC have already developed tumor metastasis, the primary cause of mortality in patients with $\mathrm{CRC}$, at the time of diagnosis (2). Metastasis is a multistep process that remains poorly understood (3). Therefore, it is necessary to reveal the pathological mechanisms underpinning

Correspondence to: Dr Jinlong Yu, Department of General Surgery, Zhujiang Hospital of Southern Medical University, 253 Gongye Road, Guangzhou, Guangdong 510530, P.R. China E-mail: yujinlong640506@163.com

Key words: sterol regulatory element-binding protein 1, c-Myc, colorectal cancer, epithelial-mesenchymal transition, SNAIL this process and to identify the critical pathways for metastasis in CRC.

The sterol regulatory element-binding protein 1 (SREBP1) is a basic helix-loop-helix leucine zipper transcription factor involved in regulating lipid homeostasis. High SREBP1 activity is an important feature of cancer metabolic reprogramming. Previous studies have suggested that SREBP1 is an attractive target for numerous types of cancer, including breast, ovarian and pancreatic cancer $(4,5)$. The expression of SREBP1 is elevated in several types of cancer, and the inhibition of SREBP1 may inhibit cancer cell growth, migration and invasion (6,7). However, the role served by SREBP1 in CRC remains to be elucidated.

The present study demonstrated that SREBP1 is highly expressed in $\mathrm{CRC}$ in order to promote the migration and invasion in CRC cells. Furthermore, it was revealed that SREBP1 interacts with c-Myc to promote snail family transcriptional repressor 1 (SNAIL) expression, which is a key transcriptional repressor of cadherin-1 (CDH1) in epithelial-mesenchymal transition. Taken together, the results of the present study demonstrated a critical role for SREBP1 in the migration and invasion of CRC cells, providing a potential target for the treatment of CRC.

\section{Materials and methods}

Cell culture and transfection. Fresh CRC and matched adjacent non-cancerous tissues were collected from 44 patients (age range, 43-65 years; mean age, 52 year; 19 females and 25 males) who underwent surgery between January 2013 and June 2016 at the Department of General Surgery, Zhujiang Hospital of Southern Medical University (Guangzhou, China). No patients received preoperative radiotherapy, chemotherapy or had a history of any other treatment. CRC cell lines SW480, HT29, HCT116, SW620, LS180 and normal colon epithelial cells NCM460 were obtained from Foleibao Biotechnology Development Co. (Shanghai, China). All cells were cultured in Dulbecco's modified Eagle's medium (DMEM; HyClone; GE Healthcare Life Sciences, Logan, UT, USA) supplemented with $10 \%$ fetal bovine serum (FBS; Gibco; Thermo Fisher Scientific, Inc., Waltham, MA, USA), and were incubated at $37^{\circ} \mathrm{C}$ in a humidified incubator in an atmosphere of $5 \% \mathrm{CO}_{2}$. Transfection of SREBP1 (cloned into pcDNA3.1 vector; Addgene, Inc., Cambridge, MA, USA), small interfering RNA 
(siRNA) of siSREBP1 and siSNAIL (IGE Biotechnology Co., Guangzhou, China; http://www.igebio.com) were performed using Lipofectamine 2000 reagent (Thermo Fisher Scientific, Inc.), according to the manufacturer's protocol. Empty pcDNA3.1 vectors and scramble siRNA, a functional non-targeting siRNA provided by the same company (IGE Biotechnology Co, Guangzhou, China, http://www.igebio. com), were used as the negative controls. The SREBP1 siRNA sequence was as follows: 5'-GCUCCUCACUUGAAGGCU UTT-3'. SNAIL siRNA sequence was as follows: 5'-CCAUGA GGAGUACUGCCAATT-3'. Briefly, in a 6-well plate, $5 \mu \mathrm{g}$ SREBP1 plasmid, $200 \mathrm{~nm}$ siSREBP1 and $200 \mathrm{~nm}$ siSNAIL were used for transfection. A total of $48 \mathrm{~h}$ after transfection, the cells were collected and prepared for analysis.

Reverse transcription-quantitative polymerase reaction $(R T-q P C R)$. Total RNA was extracted using TRIzol ${ }^{\circledR}$ reagent (Invitrogen; Thermo Fisher Scientific, Inc.) and was reverse-transcribed into cDNA at $42^{\circ} \mathrm{C}$ for $1 \mathrm{~h}$ using Premix Ex Taq (Takara Biotechnology Co., Ltd., Dalian, China). cDNA was analyzed using the CFX96 Real-Time system (Bio-Rad Laboratories, Inc., Hercules, CA, USA). The PCR thermocycling conditions were as follows: Initial denaturation at $95^{\circ} \mathrm{C}$ for $3 \mathrm{~min}$, followed by 30 cycles at $95^{\circ} \mathrm{C}$ for $15 \mathrm{sec}$, $60^{\circ} \mathrm{C}$ for $30 \mathrm{sec}$ and $72^{\circ} \mathrm{C}$ for $1 \mathrm{~min}$. Gene expression was calculated using the $2^{-\Delta \Delta \mathrm{Cq}}$ method for relative quantification (8). GAPDH was used as endogenous reference gene. Primers used were as follows: GAPDH forward, GGAGCG AGATCCCTCCAAAA and reverse, GGCTGTTGTCATACT TCTCATGG; SREBP1 forward, ACAGTGACTTCCCTG GCCTAT and reverse, GCATGGACGGGTACATCTTCAA; c-Myc forward, GGCTCCTGGCAAAAGGTCA and reverse, CTGCGTAGTTGTGCTGATGT; CDH2 forward, TCAGGC GTCTGTAGAGGCTT and reverse, ATGCACATCCTTCGA TAAGACTG; fibronectin-1 (FN1) forward, CGGTGGCTG TCAGTCAAAG and reverse, AAACCTCGGCTTCCTCCA TAA; vimentin (VIM) forward, GACGCCATCAACACC GAGTT and reverse, CTTTGTCGTTGGTTAGCTGGT; SNAIL forward, TCGGAAGCCTAACTACAGCGA and reverse, AGATGAGCATTGGCAGCGAG; and Twist-related protein 1 (TWIST1) forward, GTCCGCAGTCTTACGAGG AG and reverse, GCTTGAGGGTCTGAATCTTGCT. All procedures were performed in accordance with the ethical standards of the Research Committee of Zhujiang Hospital of Southern Medical University and with the 1964 Declaration of Helsinki and its later amendments. Written informed consent was obtained from all individual participants included in the study.

Western blot analysis. Proteins from the nucleus and cytoplasm were separated using the Nuclear and Cytoplasmic Protein Extraction kit (Beyotime Institute of Biotechnology, Haimen, China). The protein concentration was then determined using the BCA Protein Assay kit (Beyotime Institute of Biotechnology). Briefly, the absorbance was measured at $562 \mathrm{~nm}$ and protein concentration was determined relative to bovine serum albumin. The protein concentration was calculated from the standard curve: Protein concentration sample $_{\text {e }}=2.624 \mathrm{x}\left(\right.$ OD562 sample $^{-}$ OD562 $\left.2_{\text {blank }}\right)(\mu \mathrm{g} / \mu \mathrm{l})$. Then, $\sim 20 \mu \mathrm{g}$ of protein per lane was separated by $12 \%$ SDS-PAGE and proteins were transferred onto polyvinylidene difluoride membranes (EMD Millipore, Billerica, MA, USA). Subsequent to blocking the membranes with $5 \%$ milk at room temperature for $1 \mathrm{~h}$, membranes were subsequently incubated with primary antibodies against the following: SREBP1 (1:1,000; cat no. ab57999; Abcam, Cambridge, UK), $\beta$-actin (1:5,000; cat no. 4967; Cell Signaling Technology, Inc., Danvers, MA, USA), histone H3 (1:5,000; cat no. 9715; Cell Signaling Technology, Inc.). SNAIL (1:500; cat no. C15D3; Cell Signaling Technology, Inc.) at $4^{\circ} \mathrm{C}$ overnight. $\beta$-actin served as the control for total protein. Histone H3 served as the control for nuclear proteins. Secondary antibody horseradish peroxidase-conjugated mouse/rabbit IgG (1:5,000 dilution; cat no. 12971 and 12972; Beyotime Institute of Biotechnology), were then incubated with the membranes at room temperature for $1 \mathrm{~h}$. The membranes were subsequently exposed to X-ray film (Beyotime Institute of Biotechnology).

Cell migration and invasion assay. For the wound healing assay, $5 \times 10^{4} \mathrm{CRC}$ cells were cultured on 6 -well plates for $24 \mathrm{~h}$ prior to a 1-mm wide scratch being applied to the bottom of the plate. The recovery of the scratch wound was monitored $24 \mathrm{~h}$ later using a light microscope (magnification, $\mathrm{x} 10$ ). The cell-free wound area was measured using ImageJ software (1.41v, National Institutes of Health, Bethesda, MD, USA) and the migration rate was determined by quantifying the wound closure area. For the invasion assay, $10 \mathrm{ml}$ Matrigel (BD Biosciences, Franklin Lakes, NJ, USA) was dissolved in $50 \mathrm{ml}$ serum-free DMEM (HyClone; GE Healthcare Life Sciences) and applied to the upper chamber of 8-mm pore-size polycarbonate membrane filters (Corning Incorporated, Corning, NY, USA), and placed in a $37^{\circ} \mathrm{C}$ incubator for $5 \mathrm{~h}$ prior to seeding the cells. CRC cells were seeded with serum-free DMEM in the upper chamber at $1 \times 10^{5}$ cells/well, and the bottom chamber of the apparatus contained DMEM with 10\% FBS (Gibco; Thermo Fisher Scientific, Inc.). Transwell inserts were then incubated for $48 \mathrm{~h}$ at $37^{\circ} \mathrm{C}$. Following incubation, the invaded cells that had invaded through the pores of the filter and into the lower chamber were fixed with $70 \%$ methanol for $10 \mathrm{~min}$ at room temperature and stained with $1 \%$ toluidine blue (Beyotime Institute of Biotechnology) for $15 \mathrm{~min}$ at room temperature. Cell numbers were counted in ten randomly selected microscopic fields per membrane using a light microscope (magnification, x100).

Chromatin immunoprecipitation (ChIP). ChIP experiments were performed using a SimpleChIP ${ }^{\circledR}$ Plus Sonication Chromatin IP kit (Cell Signaling Technology, Inc.), according to the manufacturer's protocol. Normal rabbit IgG antibody was used as a control. Antibodies used were as follows: Normal rabbit IgG (1:500 dilution; cat. no. 2729; Cell Signaling Technology, Inc.) and anti-c-Myc (1:500 dilution; cat. no. 13978; Cell Signaling Technology, Inc.) at $4^{\circ} \mathrm{C}$ overnight. Subsequent qPCR was performed using the CFX96 Real-Time system (Bio-Rad Laboratories, Inc.). Data was calculated using the $2^{-\Delta \Delta C q}$ method for relative quantification (8). The PCR thermocycling conditions were as follows: Initial denaturation at $95^{\circ} \mathrm{C}$ for $3 \mathrm{~min}$, followed by 30 cycles at $95^{\circ} \mathrm{C}$ for $15 \mathrm{sec}, 60^{\circ} \mathrm{C}$ for $30 \mathrm{sec}$ and $72^{\circ} \mathrm{C}$ for $1 \mathrm{~min}$. Primers for the SNAIL promoter region were as follows: Forward, 3'-CTTGACTCAGTGTCCCTCC-5' and reverse, 3'-GCCAGAACTAATCGCATC-5'. 
A

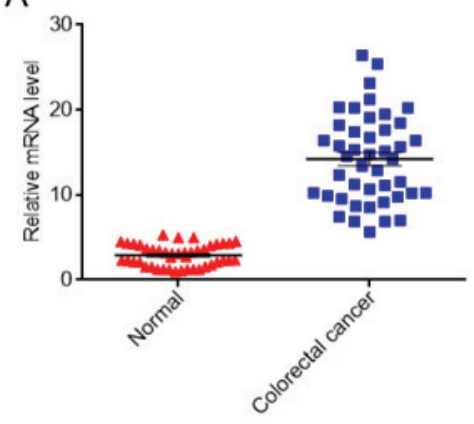

B

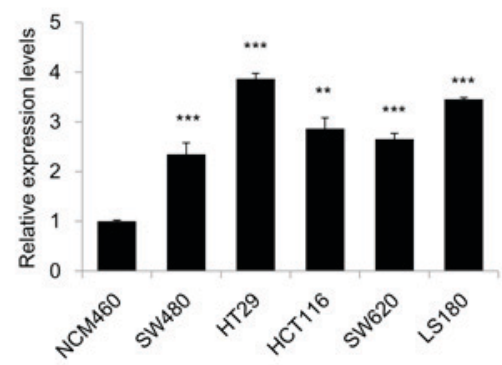

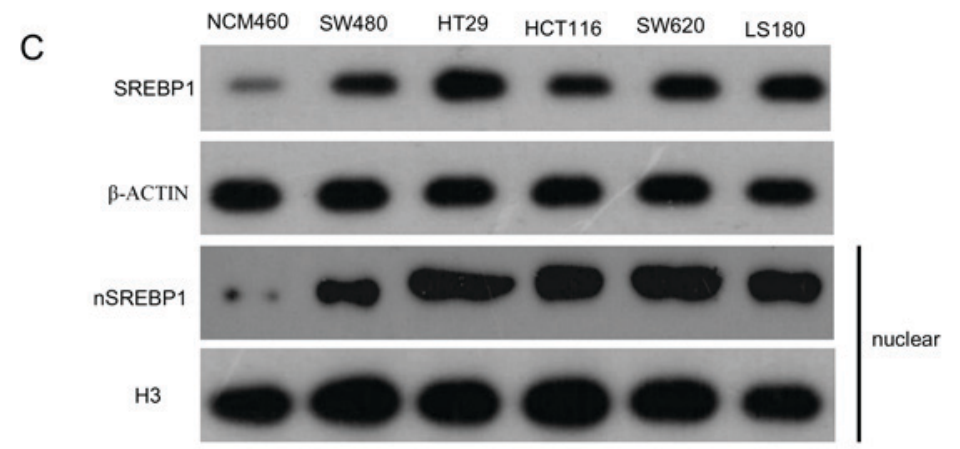

Figure 1. SREBP1 is highly expressed in colorectal cancer tissues and cell lines. (A) qPCR analysis of SREBP1 mRNA expression in 44 paired CRC samples and adjacent normal tissues. (B) qPCR analysis of the SREBP1 mRNA level in different CRC cell lines and a normal control cell line. Data are presented as the mean \pm standard deviation of three independent experiments. ${ }^{* *}<0.01$ and ${ }^{* * *} \mathrm{P}<0.001$ vs. NCM460. (C) Western blot analysis of the expression of total and nuclear SREBP1 in different CRC cell lines and a normal control cell line. $\beta$-actin served as the control for total protein and histone $\mathrm{H} 3$ served as the control for nuclear protein. SREBP1, sterol regulatory element-binding protein 1; qPCR, quantitative polymerase chain reaction; CRC, colorectal cancer; H3, histone H3.

Co-immunoprecipitation (Co-IP). Transfected CRC cells were lysed in cell lysis buffer [50 mM Tris- $\mathrm{HCl}$ (pH 7.4), $150 \mathrm{mM}$ $\mathrm{NaCl}, 1 \mathrm{mM}$ EDTA, 1\% NP40, protease inhibitors] for $30 \mathrm{~min}$. Lysates were incubated with Proteins $\mathrm{A}$ and $\mathrm{G}$ (Invitrogen; Thermo Fisher Scientific, Inc.) at $4^{\circ} \mathrm{C}$ overnight, which were coupled with c-Myc antibodies overnight at $4^{\circ} \mathrm{C}$. Following immunoprecipitation, Proteins $\mathrm{A}$ and $\mathrm{G}$ was washed with lysis buffer five times to remove any un-precipitated proteins and were boiled in SDS buffer for $5 \mathrm{~min}$ to elute the proteins. The eluent was analyzed for precipitated SREBP1 protein using western blot analysis as aforementioned. A normal IgG antibody was used as a control. Antibodies against the following were used: SREBP1 (1:500 dilution, cat. no. 28481; Abcam), c-Myc (1:100 dilution, cat. no. 13978; Cell Signaling Technology, Inc.) and normal rabbit IgG (1:1,000 dilution, cat. no. 2729; Cell Signaling Technology, Inc.).

Statistical analysis. Data are presented as the mean \pm SD. Statistical comparisons were performed using unpaired two-tailed Student's t-tests or one-way analysis of variance followed by a Newman-Keuls test. $\mathrm{P} \leq 0.05$ was considered statistically significant.

\section{Results}

SREBP1 is highly expressed in colorectal cancer tissues and cell lines. To evaluate the potential roles for SREBP1 in CRC, the expression of SREBP1 in 44 paired CRC samples and adjacent normal tissues were analyzed using RT-qPCR. The results of the present study demonstrated that SREBP1 is highly expressed in CRC samples, compared with expression in healthy controls (Fig. 1A). The expression of SREBP1 was also measured in several CRC cell lines, including SW480, HT29, HCT116, SW620 and LS180, and a higher expression of SREBP1 was observed in these CRC cell lines compared with normal colon epithelial cells (NCM460; Fig. 1B). Additionally, western blot analysis of total and nuclear SREBP1 further confirmed that SREBP1 is highly expressed and activated in these CRC cell lines (Fig. 1C), suggesting a potential role for SREBP1 in colorectal cancer.

SREBPI regulates the proliferation, migration and invasion in CRC cells. SREBP1 is a well-known key regulator of lipid metabolism. Recently, growing evidence has indicated that SREBP1 is involved in cancer development and progression through targeting lipid metabolism and cancer cell migration. To determinate the effects of SREBP1 on the growth of CRC cells, the proliferation rate of CRC SW480 cells upon SREBP1 overexpression or SREBP1 knockdown was measured. It was revealed that SREBP1 overexpression increased the proliferation rate of CRC cells, while SREBP1 knockdown using siRNA decreased the proliferation ability of CRC cells (Fig. 2A). These results suggested that SREBP1 may regulate CRC cell growth.

As metastasis is a major problem in CRC and is associated with cancer cell migration and invasion (9-11), the present study aimed to determine whether SREBP1 may affect the migration or invasion of CRC cells. Wound healing assays were performed and it was revealed that SREBP1 overexpression significantly increased the wound closure area, suggesting 

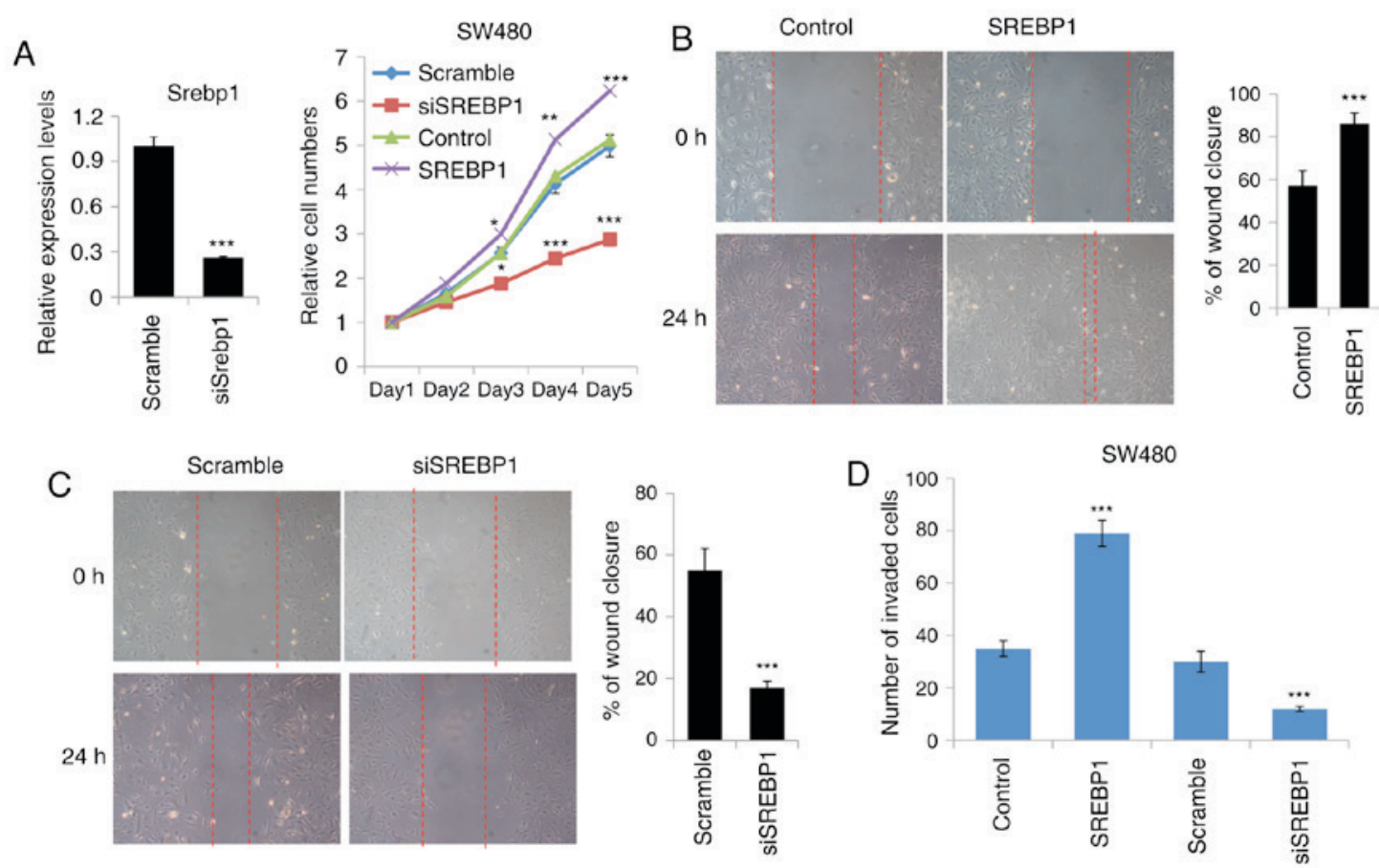

Figure 2. SREBP1 regulates the proliferation, migration and invasion of CRC cells. (A) Cell proliferation assays of CRC cells transfected with SREBP1 or SREBP1 siRNA. Right, knockdown efficiency of siSREBP1 and left, cell proliferation assays. Data are presented as the mean \pm standard deviation of three independent experiments. "P<0.05, ${ }^{* *} \mathrm{P}<0.01$ and ${ }^{* * * *} \mathrm{P}<0.001$ vs. scramble or control. (B) Wound healing assays of CRC cells transfected with SREBP1. Right, representative image of wound healing assays and left, analysis of the migration rate. The migration rate was determined by quantifying the wound closure area after $24 \mathrm{~h}$ of migration. Data are presented as the mean \pm standard deviation of three independent experiments. ${ }^{* * * *} \mathrm{P}<0.001$ vs. control. (C) Wound healing assays of CRC cells transfected with SREBP1 siRNA. Right, representative image of wound healing assays and left, analysis of the migration rate. The migration rate was determined by quantifying the wound closure area after $24 \mathrm{~h}$ of migration. Data are presented as the mean \pm standard deviation of three independent experiments. ${ }^{* * * *} \mathrm{P}<0.001$ vs. scramble. (D) Transwell assays of CRC cells transfected with SREBP1 or SREBP1 siRNA. Cells that had spread through the pores of the filter and into the lower chamber were fixed with $70 \%$ methanol and were counted. Data are presented as the mean \pm standard deviation of three independent experiments. ${ }^{* * * *} \mathrm{P}<0.001$ vs. scramble or control. SREBP1, sterol regulatory element-binding protein 1; CRC, colorectal cancer; si, small interfering RNA.

that SREBP1 may accelerate the migration rate in CRC cells (Fig. 2B). However, knockdown of SREBP1 using siRNA resulted in impairment of the migration ability in CRC cells (Fig. 2C). Furthermore, Transwell assays were performed in order to confirm the effects of SREBP1 on CRC cell migration. Compared with the control vector-expressing cells, SREBP1-overexpressing cells exhibited a 2 -fold increase in the number of cells penetrating the Transwell membrane (Fig. 2D). In line with this, SREBP1 knockdown reduced the number of cells penetrating the membrane, indicating important roles for SREBP1 in CRC cell invasion (Fig. 2D). Taken together, these data demonstrated that SREBP1 may promote $\mathrm{CRC}$ cell growth, migration and invasion.

SREBP1 promotes EMT and migration by increasing SNAIL expression. Given that EMT is the key process involved incancer migration and that SREBP1 has been reported to serve a role in breast cancer migration (6), it is reasonable to hypothesize that SREBP1 promotes CRC cell migration through EMT. In order to elucidate the mechanism by which SREBP1 promotes CRC cell migration, the expression of several mesenchymal genes, including CDH2, FN1, VIM, SNAIL and TWIST1, was measured. Data obtained through qPCR demonstrated that overexpression of SREBP1 increased the expression of all these mesenchymal genes, while knockdown of SREBP1 using siRNA inhibited these genes (Fig. 3A and B). Furthermore, the change in SNAIL expression was most marked upon SREBP1 overexpression or knockdown (Fig. 3A and B). Western blot analysis of SNAIL revealed similar results (Fig. 3C). This led to the hypothesis that SNAIL is responsible for the SREBP1-driven EMT in CRC cells. In order to confirm this, wound healing and Transwell assays were performed in CRC cells transfected with SNAIL siRNA or SREBP1 plus SNAIL siRNA. Wound healing assays demonstrated that SREBP1 does not promote CRC cell migration when SNAIL is inhibited by siRNA (Fig. 3D and E), suggesting that the function of SREBP1 is SNAIL-dependent. Transwell assays further confirmed the results of the wound healing assays (Fig. 3D). Taken together, these results indicated that SREBP1 promotes migration and ETM by increasing SNAIL expression.

SREBP1 facilitates the binding of $c-M y c$ to the SNAIL promoter. Due to the fact that c-Myc is highly expressed in CRC and that SNAIL is one of the crucial targets of c-Myc in EMT (12-14), the present study investigated whether or not c-Myc is responsible for SREBP1-driven SNAIL expression and EMT in CRC. To begin with, the expression of c-Myc was measured in different CRC cell lines and it was revealed that c-Myc is highly expressed in all these CRC cell lines (Fig. 4A), consistent with the results of previous studies (12-14). As SREBP1 has been reported to interact with c-Myc to enhance the function of c-Myc in somatic cell 
A

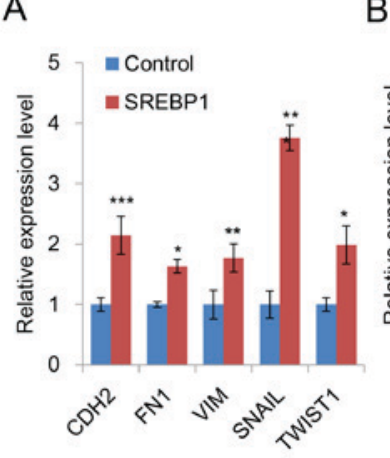

D

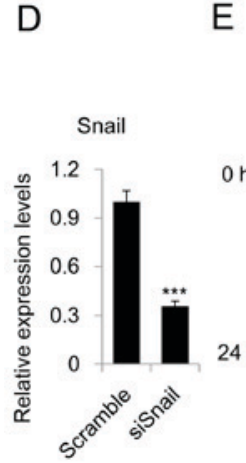

B

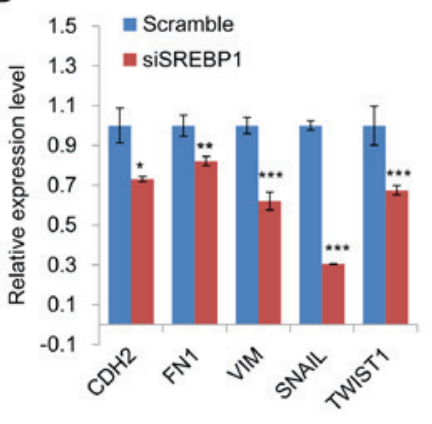

C

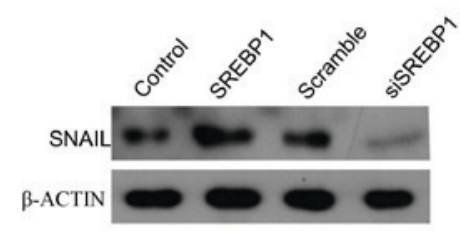

$\mathrm{F}$

SW480

Control+siSNAIL SREBP1+siSNAIL
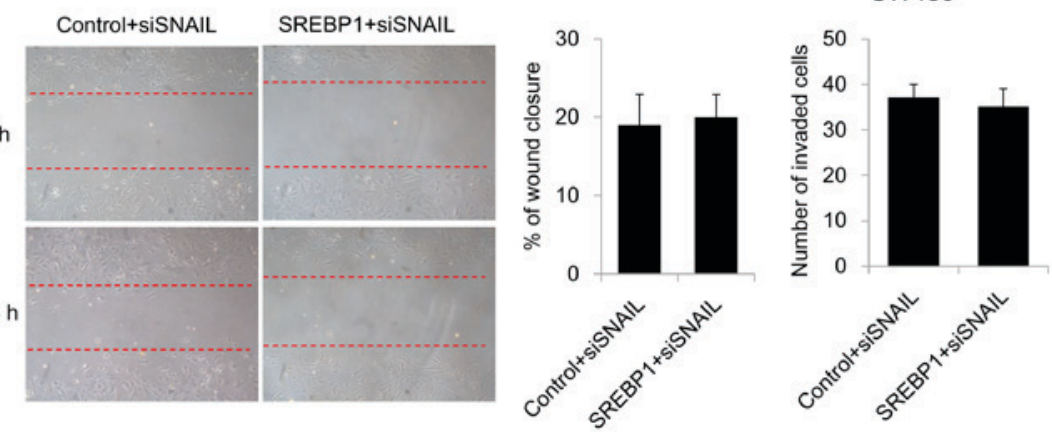

Figure 3. SREBP1 promotes EMT by increasing SNAIL expression. (A) qPCR analysis of the expression of EMT-related genes in CRC cells transfected with SREBP1 or a control vector. Data are presented as the mean \pm standard deviation of three independent experiments. " $\mathrm{P}<0.05,{ }^{* * *} \mathrm{P}<0.01$ and ${ }^{* * * *} \mathrm{P}<0.001$ vs. control. (B) qPCR analysis of the expression of EMT-related genes in CRC cells transfected with SREBP1 siRNA or scramble siRNA. Data are presented as the mean \pm standard deviation of three independent experiments. ${ }^{*} \mathrm{P}<0.05,{ }^{* *} \mathrm{P}<0.01$ and ${ }^{* * *} \mathrm{P}<0.001$ vs. scramble. (C) Western blot analysis of SNAIL expression in CRC cells transfected with control vector, SREBP1, scramble or siSREBP1. (D) qPCR analysis of the knockdown efficiency of siSNAIL. Data are presented as the mean \pm standard deviation of three independent experiments. ${ }^{* * *} \mathrm{P}<0.001$ vs. scramble. (E) Wound healing assays of CRC cells transfected with SREBP1 and SNAIL siRNA. Right, representative image of wound healing assays and left, analysis of the migration rate. The migration rate was determined by quantifying the wound closure area after $24 \mathrm{~h}$ of migration. Data are presented as the mean \pm standard deviation of three independent experiments. (F) Transwell assays of CRC cells transfected with SREBP1 and SNAIL siRNA. Cells that had invaded through the pores of the filter and into the lower chamber were fixed with $70 \%$ methanol and counted. Data are presented as the mean \pm standard deviation of three independent experiments. SREBP1, sterol regulatory element-binding protein 1; EMT, epithelial-mesenchymal transition; qPCR, quantitative polymerase chain reaction; CRC, colorectal cancer; si, small interfering RNA; CDH2, cadherin 2; FN1, fibronectin-1; VIM, vimentin; TWIST1, Twist-related protein 1.

A

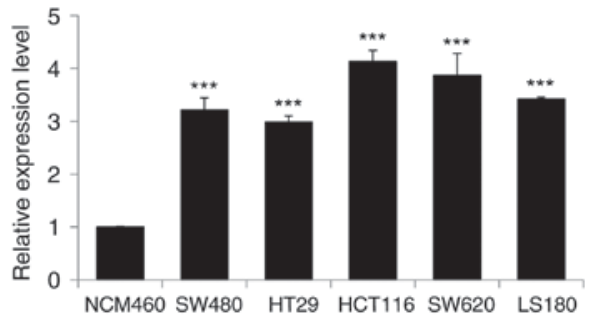

C

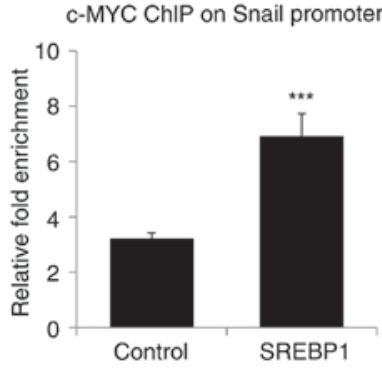

B

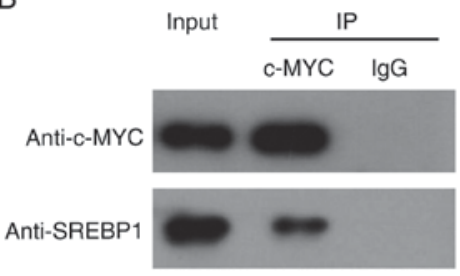

$\mathrm{D}$

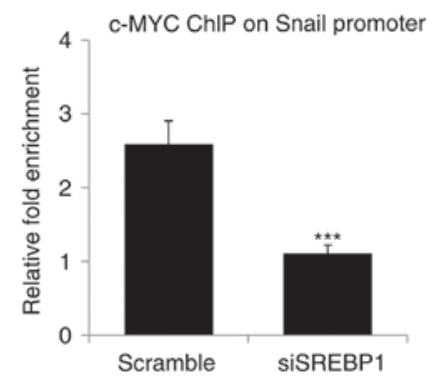

Figure 4. SREBP1 facilitates the binding of c-Myc to the SNAIL promoter. (A) qPCR analysis of the expression of c-Myc in different CRC cell lines and a normal cell line. Data are presented as the mean \pm standard deviation of three independent experiments. ${ }^{* * * *} \mathrm{P}<0.001$ vs. scramble or control. (B) Co-IP of SREBP1 and c-Myc in CRC cells transfected with SREBP1 and c-Myc. (C) ChIP-qPCR analysis of the binding of c-Myc on the SNAIL promoter in CRC cells transfected with SREBP1. Data are presented as the mean \pm standard deviation of three independent experiments. ${ }^{* * *} \mathrm{P}<0.001$ vs. control. (D) ChIP-qPCR analysis of the binding of c-Myc to the SNAIL promoter in CRC cells transfected with SREBP1 siRNA and scramble siRNA. Data are presented as the mean \pm standard deviation of three independent experiments. ${ }^{* * *} \mathrm{P}<0.001$ vs. scramble. SREBP1, sterol regulatory element-binding protein 1 ; qPCR, quantitative polymerase chain reaction; CRC, colorectal cancer; Co-IP, co-immunoprecipitation; ChIP, chromatin immunoprecipitation; siRNA, small interfering RNA; IgG, immunoglobulin G. 
reprogramming (15), we hypothesized that there may be an associated between SREBP1 and c-Myc in CRC. In the present study, Co-IP was performed, revealing that SREBP1 interacts with c-Myc in CRC cells (Fig. 4B). Due to the fact that c-Myc is involved in EMT through direct targeting of the promoter of SNAIL $(16,17)$, ChIP-qPCR was performed in order to analyze the binding of c-Myc to the SNAIL promoter in CRC cells transfected with SREBP1 or siSREBP1 and corresponding control vectors. ChIP-qPCR revealed a higher enrichment of c-Myc on the SNAIL promoter when SREBP1 was overexpressed in CRC cells (Fig. 4C). Knockdown of SREBP1 led to a decreased binding of c-MYC to the SNAIL promoter (Fig. 4D). Therefore, the results of the present study demonstrated that SREBP1 facilitates the binding of c-Myc to the SNAIL promoter in CRC cells, thereby accelerating SNAIL expression, EMT and migration.

\section{Discussion}

Metastasis is the primary cause of mortality in patients with CRC, the mechanism of which remains to be fully elucidated $(3,18)$. Further investigation into the underlying mechanisms of CRC metastasis is required. Emerging evidence has indicated that SREBP1, a master transcription factor that controls metabolic reprogramming in cancer cells, is also involved in the invasion and migration of cancer cells (19). However, the roles of SREBP1 in CRC remain unclear. The present study demonstrated that SREBP1 is highly expressed in CRC tissues and cell lines, suggesting a potential role for SREBP1 in CRC. Furthermore, it was revealed that SREBP1 regulates CRC cell proliferation, migration and invasion through increasing SNAIL expression and accelerating EMT. Finally, mechanistic studies revealed that SREBP1-driven EMT depends upon c-Myc, as SREBP1 interacts with c-Myc to enhance its binding to the SNAIL promoter, thereby increasing SNAIL expression and subsequent EMT and cell migration.

Metabolic reprogramming is one of the key features of cancer cells (18). SREBP1 has become an area of interest in cancer biology because it is a key regulator of lipid metabolism (19). Recently, the high expression level of SREBP1 in cancer cells has encouraged novel research $(4,5,7,20)$. The majority of these studies have focused on the impact of SREBP1 on cancer cell metabolism, particularly lipid synthesis and desaturation $(4,5,7,20)$. Few previous studies have demonstrated that SREBP1 is involved in cell migration and invasion (6), and the underlying mechanism of this remains unclear. However, the present study demonstrated that SREBP1 regulates the migration and invasion of CRC cells. Furthermore, the mechanism through which SREBP1 promoting EMT was also investigated, and it was revealed that SREBP1 interacts with c-Myc to increase the expression of SNAIL, thereby promoting EMT in CRC.

The proto-oncogene, $\mathrm{c}-\mathrm{Myc}$, serves crucial roles in various types of cancer and is known to be highly expressed in the majority of cases of CRCs (12-14,21-23). It has been reported that c-Myc is primarily involved in regulation at the transcriptional and post-transcriptional levels in multiple types of cancer (14,21-23). However, the regulatory mechanisms of c-Myc in CRC remain unclear. The present study not only revealed a synergistic as association between SREBP1 and c-Myc, which was consistent with the observations of previous studies (15), but also provided a novel regulatory model for the regulation of c-Myc in $\mathrm{CRC}$.

To conclude, the results of the present study demonstrated that SREBP1 interacts with c-Myc to enhance the EMT-promoting function of c-Myc. The present study identified a novel role for SREBP1 in the metastasis of CRC cells and provided insight on the regulatory mechanism of c-Myc.

\section{Acknowledgements}

The authors would like to thank the Department of General Surgery, Zhujiang Hospital of Southern Medical University (Guangzhou, China) of Professor Jinglong Yu for providing technical support.

\section{References}

1. Weitz J, Koch M, Debus J, Hohler T, Galle P and Buchler MW: Colorectal cancer. Lancet 365: 153-165, 2005.

2. Hu CE and Gan J: TRIM37 promotes epithelial-mesenchymal transition in colorectal cancer. Mol Med Rep 15: 1057-1062, 2017.

3. Boutin AT, Liao WT, Wang M, Hwang SS, Karpinets TV, Cheung H, Chu GC, Jiang S, Hu J, Chang K, et al: Oncogenic Kras drives invasion and maintains metastases in colorectal cancer. Genes Dev 31: 370-382, 2017.

4. Williams KJ, Argus JP, Zhu Y, Wilks MQ, Marbois BN, York AG, Kidani Y, Pourzia AL, Akhavan D, Lisiero DN, et al: An essential requirement for the SCAP/SREBP signaling axis to protect cancer cells from lipotoxicity. Cancer Res 73: 2850-2862, 2013.

5. Griffiths B, Lewis CA, Bensaad K, Ros S, Zhang Q, Ferber EC, Konisti S, Peck B, Miess H, East P, et al: Sterol regulatory element binding protein-dependent regulation of lipid synthesis supports cell survival and tumor growth. Cancer Metab 1: 3, 2013.

6. Bao J, Zhu L, Zhu Q, Su J, Liu M and Huang W: SREBP-1 is an independent prognostic marker and promotes invasion and migration in breast cancer. Oncol Lett 12: 2409-2416, 2016.

7. Li W, Tai Y, Zhou J, Gu W, Bai Z, Zhou T, Zhong Z, McCue PA, Sang N, Ji JY, et al: Repression of endometrial tumor growth by targeting SREBP1 and lipogenesis. Cell cycle 11: 2348-2358, 2012.

8. Livak KJ and Schmittgen TD: Analysis of relative gene expression data using real-time quantitative PCR and the 2(-Delta Delta C(T)) method. Methods 25: 402-408, 2001.

9. Jackstadt R, Röh S, Neumann J, Jung P, Hoffmann R, Horst D, Berens C, Bornkamm GW, Kirchner T, Menssen A and Hermeking H: AP4 is a mediator of epithelial-mesenchymal transition and metastasis in colorectal cancer. J Exp Med 210: 1331-1350, 2013.

10. Kurita K, Maeda M, Mansour MA, Kokuryo T, Uehara K, Yokoyama Y, Nagino M, Hamaguchi M and Senga T: TRIP13 is expressed in colorectal cancer and promotes cancer cell invasion. Oncol Lett 12: 5240-5246, 2016.

11. Long J, Xie Y, Yin J, Lu W and Fang S: SphK1 promotes tumor cell migration and invasion in colorectal cancer. Tumour Biol 37: 6831-6836, 2016.

12. Boudjadi S, Carrier JC, Groulx JF and Beaulieu JF: Integrin $\alpha 1 \beta 1$ expression is controlled by c-MYC in colorectal cancer cells. Oncogene 35: 1671-1678, 2016.

13. Zhang L, Liu X, Zuo Z, Hao C and Ma Y: Sphingosine kinase 2 promotes colorectal cancer cell proliferation and invasion by enhancing MYC expression. Tumour Biol 37: 8455-8460, 2016.

14. Liu Z, Jiang Y, Hou Y, Hu Y, Cao X, Tao Y, Xu C, Liu S, Wang S, Wang L, et al: The IкB family member Bcl-3 stabilizes c-Myc in colorectal cancer. J Mol Cell Biol 5: 280-282, 2013.

15. Wu Y, Chen K, Liu X, Huang L, Zhao D, Li L, Gao M, Pei D, Wang C and Liu X: Srebp-1 Interacts with c-Myc to enhance somatic cell reprogramming. Stem cells 34: 83-92, 2016.

16. Smith AP, Verrecchia A, Fagà G, Doni M, Perna D, Martinato F, Guccione E and Amati B: A positive role for Myc in TGFbeta-induced Snail transcription and epithelial-to-mesenchymal transition. Oncogene 28: 422-430, 2009. 
17. Cho KB, Cho MK, Lee WY and Kang KW: Overexpression of c-myc induces epithelial mesenchymal transition in mammary epithelial cells. Cancer Lett 293: 230-239, 2010.

18. Yao C, Su L, Shan J, Zhu C, Liu L, Liu C, Xu Y, Yang Z, Bian X, Shao J, et al: IGF/STAT3/NANOG/slug signaling axis simultaneously controls epithelial-mesenchymal transition and stemness maintenance in colorectal cancer. Stem cells 34: 820-831, 2016.

19. Beloribi-Djefaflia S, Vasseur S and Guillaumond F: Lipid metabolic reprogramming in cancer cells: Oncogenesis 5: e189, 2016.

20. Sun Y, He W, Luo M, Zhou Y, Chang G, Ren W, Wu K, Li X, Shen J, Zhao X and $\mathrm{Hu}$ Y: SREBP1 regulates tumorigenesis and prognosis of pancreatic cancer through targeting lipid metabolism. Tumour Biol 36: 4133-4141, 2015.
21. Chuan YC, Iglesias-Gato D, Fernandez-Perez L, Cedazo-Minguez A, Pang ST, Norstedt G, Pousette A and Flores-Morales A: Ezrin mediates c-Myc actions in prostate cancer cell invasion. Oncogene 29: 1531-1542, 2010.

22. Zhu W, Cai MY, Tong ZT, Dong SS, Mai SJ, Liao YJ, Bian XW, Lin MC, Kung HF, Zeng YX, et al: Overexpression of EIF5A2 promotes colorectal carcinoma cell aggressiveness by upregulating MTA1 through C-myc to induce epithelial-mesenchymaltransition. Gut 61: 562-575, 2012.

23. Mansour MA, Hyodo T, Akter KA, Kokuryo T, Uehara K, Nagino $M$ and Senga T: SATB1 and SATB2 play opposing roles in c-Myc expression and progression of colorectal cancer. Oncotarget 7: 4993-5006, 2016 